\title{
Beeinflusst die Kühlsprayanwendung am Fuß die dynamische Stabilisationsfähigkeit?
}

\section{Short-Term Cryotherapy - Putting Dynamic Foot and Ankle Stability at Risk?}

\author{
Autoren \\ Institute \\ C. Huber ${ }^{1,2}$, J. Swanenburg ${ }^{1,3,4}$, M. Leusch², P. M. Tscholl ${ }^{5}$ \\ Departement Physiotherapie, Universitätsklinik Balgrist, Zürich, Schweiz \\ Zürcher Fachhochschule für Angewandte Wissenschaften ZHAW, Winterthur, Schweiz \\ Departement für Chiropraktik, Universität Zürich, Universitätsklinik Balgrist, Zürich, Schweiz \\ ${ }^{4}$ Forschung Physiotherapie Ergotherapie, Direktion Forschung und Lehre, Universitätsspital Zürich, Schweiz \\ Departement Orthopädie, Universität Zürich, Universitätsklinik Balgrist, Zürich, Schweiz
}

\author{
Schlüsselwörter \\ - Kryotherapie \\ - Sport \\ - Sprunggelenk \\ - Kraftmessplatte \\ - Propriozeption \\ Key words \\ - cryotherapy \\ - ice spray \\ - ankle \\ - force plate \\ - proprioception
}

Bibliografie

DOI http://dx.doi.org/

10.1055/s-0034-1399100

Sportverl Sportschad 2015; 29 :

99-106 @ Georg Thieme Verlag

KG Stuttgart · New York .

ISSN 0932-0555

Korrespondenzadresse

Corinne Huber

Medbase Winterthur

Brunngasse 6

8400 Winterthur

huber_corinne@hotmail.com

\section{Zusammenfassung \\ $\nabla$}

Hintergrund: Kälte wird bei der Akutbehandlung von muskuloskelettalen Verletzungen am Spielfeldrand aufgrund ihrer analgetischen Wirkung und der einfachen Anwendung häufig eingesetzt. Nicht selten kehren die Athleten direkt nach der Intervention ins Spielgeschehen zurück. Ob die kurzzeitige Kühlung wie bei der Anwendung von Kühlspray einen Einfluss auf die dynamische Stabilisationsfähigkeit besitzt, ist nicht bekannt. Ziel dieser Studie war herauszufinden, ob die Kühlsprayanwendung am Fuss die dynamische Stabilisationsfähigkeit gesunder Probanden beeinflusst. Methode: Es wurde eine randomisiert kontrollierte und einfach verblindete Pilotstudie im Crossoverdesign (intra-individueller Vergleich) durchgeführt. Verglichen wurde die Time-to-stability (TTS) in mediolaterale (ML) und anteroposteriore (AP) Richtung von 22 gesunden sportlichen Probanden (15 Frauen, 7 Männer im Alter von 31,8 Jahren $\pm 5,7$ ), gemessen mit einer Kraftmessplatte bei zwei verschiedenen Tests (Side-Step-down und 1-Leg-jump). Als Intervention wurden Kühlspray und Wasserspray am lateralen oberen Sprunggelenk (OSG) verwendet. Erhoben wurde bei den Probanden Alter, Geschlecht, Größe, Gewicht, Vorverletzungen des OSG, Tegner-Aktivitätsskala und dominantes Bein.

Ergebnisse: In keinem der beiden Tests wurde ein signifikanter Unterschied der TTS zur BaselineMessung nach der Applikation von Kühlspray und Wasserspray am lateralen OSG festgestellt $(p>0,05)$. Die Überprüfung der Zusatzvariablen „Vorverletzungen des OSG“ zeigte einen signifikanten Einfluss auf die TTS am nicht dominanten Bein beim 1-Leg-jump in mediolaterale Richtung nach der Applikation von Kühlspray ( $\mathrm{p}=0,027$ ). Beim dominanten Bein zeigte sich eine Tendenz $(\mathrm{p}=0,062)$. Schlussfolgerung: Es konnte kein signifikanter Einfluss einer Kühlsprayanwendung am lateralen OSG auf die dynamische Stabilisationsfähigkeit

\section{Abstract \\ $\nabla$}

Background: The application of ice or other forms of cooling represent a common method to treat acute musculoskeletal injuries during sporting events in order to reduce pain. Often athletes return to competition immediately after cooling. It is not known if short-term cryotherapy in the form of ice spray has an influence on the joint's dynamic stability. The aim of this study was to investigate if application of ice spray to the ankle has an effect on the dynamic stability of the joint in healthy participants.

Methods: A randomized controlled single-blind pilot study with crossover-design was conducted. 22 healthy athletic participants (15 women, 7 men, mean age 31.8 years \pm 5.7 ) were included. Time-to-stability (TTS) was used to investigate the effect of the interventions, ice spray and water spray, applied on the lateral ankle. TTS was assessed in medio-lateral (ML) and antero-posterior (AP) direction on a force plate with two different tests (side step down and 1-leg jump). Collected co-variables were age, gender, height, weight, previous ankle injuries, Tegner activity scale and leg dominance.

Results: There were no significant differences between the two tests (side step down and 1-leg jump) in TTS after the application of ice spray or a water spray compared to the baseline $(p>0.05)$. There was no significant difference between the two interventions. The testing of the co-variable "previous ankle injury" showed a significant influence on the TTS in medio-lateral direction in the 1-leg jump test of the non-dominant leg after application of ice spray $(p=0.027)$. On the dominant leg same tendency could be found $(\mathrm{p}=0.062)$.

Conclusion: The application of ice spray to the lateral ankle does not have an effect on dynamic stability in healthy participants. In participants with a previous ankle injury a significant decrease in 
von gesunden Probanden gefunden werden. Bei Probanden mit einer Vorverletzung des OSG hat die Applikation von Kühlspray die dynamische Stabilisationsfähigkeit verändert. Ob weitere, die Stabilisationsfähigkeit beeinflussende Faktoren, wie Vorermüdung und Gegenspieler oder die Applikation von Kühlspray auf die Muskulatur diesen Effekt vergrößern, sollte in einer weiteren Untersuchung differenziert werden. dynamic stability after application of ice spray could be shown. Whether further factors affecting stability such as fatigue and the influence of an opponent player or the application of ice spray on adjacent muscles may augment this effect should be subject to future investigations.

\section{Einleitung}

$\nabla$

Das obere Sprunggelenk (OSG) gehört zu den am häufigsten verletzten Gelenken im Sport [1, 2], insbesondere bei Ballsportarten wie Fußball, Basketball oder Volleyball [1, 3]. Häufig werden diese Verletzungen direkt am Spielfeldrand erstversorgt. Nicht selten kehrt ein Athlet unmittelbar nach der Behandlung ins Spielgeschehen zurück. Eine aufgrund ihrer schnellen und einfachen Anwendung häufig eingesetzte Intervention in der Behandlung von akuten muskuloskelettalen Verletzungen ist die Applikation von Kälte, auch bekannt als Kryotherapie [4], was sich auf das therapeutische Absenken der lokalen Gewebstemperatur durch den Entzug von Wärme aus dem Körper bezieht [5]. Das primäre Ziel der Kälteanwendung ist eine analgetische Wirkung aufgrund abnehmender Rezeptorenempfindlichkeit und -entladungen [6, 7], verringerter Nervenleitgeschwindigkeit [8] und einem Gegenreiz zum Schmerz [7]. Ein weiterer Effekt der Kälteanwendung ist eine Reduktion der Entzündungsreaktion und des zellulären Metabolismus [6, 7].

Die durch die Kälteapplikation verminderte Nervenleitgeschwindigkeit führt einerseits zu einer Schmerzreduktion [8], andererseits zu einer möglichen Beeinflussung der efferenten motorischen Neurone, was Aspekte der Muskelfunktion wie beispielsweise Kraft, Ausdauer und Reaktionszeit beeinflussen kann [7].

Die Applikation von Kälte kann in Form von Eiswürfeln, zerstoßenem Eis, Kühlbeuteln, Kältebädern und Kühlspray erfolgen [6, 9]. Die Resultate verschiedener Studien zum Einfluss von Kälte auf die Wahrnehmung der Gelenksposition [9-11], der statischen $[3,12]$ und dynamischen Stabilisationsfähigkeit [12-15], Kraft und Geschwindigkeit [12, 16-18] sind widersprüchlich. Die Kälteanwendungen in den uns bekannten Studien fanden meist über einen Zeitraum von 15 Minuten oder länger statt. In zwei Studien hingegen wurde der Einfluss von kürzeren Eisanwendungen untersucht. Die Autoren fanden keinen Einfluss einer 10-minütigen Eisbeutelapplikation am OSG auf das statische Gleichgewicht [15], beziehungsweise einen negativen Effekt einer 10-minütigen Kühlung der ischiocruralen Muskulatur mittels Eisbeutel auf Kraft und Leistungsfähigkeit, nicht aber einer 3-minütigen Kühlung [18]. Die Arbeitsgruppe um Bleakley [6] beschäftigte sich in ihrem Übersichtsartikel mit der Frage, ob Athleten nach Kälteanwendungen ungefährdet ihre sportliche Aktivität wieder aufnehmen können. Aufgrund begrenzter Evidenz für einen nachteiligen Einfluss auf die Geschwindigkeit, Kraft sowie auf Wendigkeit basierende Laufübungen raten sie zu kurzen Kälteanwendungen sowie einem stufenweise stattfindenden Aufwärmen vor der Rückkehr in den Sport.

Insbesondere am Spielfeldrand wird als Kälteanwendung häufig Kühlspray verwendet. Nur eine der uns bekannten Studien von Surenkok et al. [10] untersuchte den Einfluss von Kühlspray auf die Wahrnehmung der Gelenksposition des Knies und auf die statische Stabilisationsfähigkeit auf einer sich bewegenden Fläche. Über die Zeitdauer der Applikation machten die Autoren keine Angaben. Die statische Stabilisationsfähigkeit wurde nicht ne- gativ beeinflusst, allerdings fanden sie einen signifikanten Unterschied in der Wahrnehmung der Gelenksposition nach der Kühlsprayapplikation. Der Einfluss auf die dynamische Stabilisationsfähigkeit wurde nicht untersucht. Da jedoch einer der häufigsten Verletzungsmechanismen am OSG die Landung in einer Inversionsposition ist [19], hat die Messung der dynamischen Stabilisationsfähigkeit einen größeren Bezug zum sportspezifischen Alltag.

Nach unserem Kenntnisstand gibt es Studien sowohl zur dynamischen Stabilisationsfähigkeit als auch zu sportspezifischen Aktivitäten $[3,12]$ nach Eisanwendungen. Diese stützen sich jedoch auf Untersuchungen von Kälteexpositionen über einen längeren Zeitraum, keine auf den Effekt nach einer kurzzeitigen Kälteintervention wie der Applikation von Kühlspray. Somit fehlen empirische Daten, um den Effekt von Kühlspray in sportspezifischen Situationen zu erforschen.

Ziel dieser Studie ist es zu untersuchen, ob die Kühlsprayanwendung am lateralen OSG einen Einfluss auf die dynamische Stabilisationsfähigkeit bei gesunden Probanden hat.

\section{Probanden/Material und Methode \\ $\nabla$}

\section{Studiendesign}

Es wurde eine randomisierte kontrollierte und einfach verblindete Pilotstudie im Crossoverdesign (intra-individueller Vergleich) durchgeführt (ClinicalTrials.gov: NCT01 961 596).

\section{Probanden und Probandenauswahl}

Für die Studienteilnahme wurden gesunde sportliche Probanden im Alter zwischen 18 und 45 Jahren mit mindestens 4/10 Punkten auf der auf Deutsch validierten Tegner-Aktivitätsskala (TAS) $[20,21]$ rekrutiert.

Ausschlusskriterien waren Verletzungen der Wirbelsäule, des Beckens oder der unteren Extremitäten, die weniger als sechs Wochen zurücklagen, eine veränderte sensorische Wahrnehmung der unteren Extremitäten, die Einnahme von das Gleichgewicht beeinflussenden Medikamenten sowie Allergien beziehungsweise Unverträglichkeiten gegenüber Kälte oder einem der Inhaltsstoffe des Kühlsprays. Die Testungen mussten schmerzfrei durchführbar sein. Die Probanden wurden vorgängig schriftlich und mündlich über den Ablauf der Studie aufgeklärt und unterschrieben eine Einverständniserklärung. Bei der kantonalen Ethikkommission Zürich wurde die Erlaubnis zur Durchführung der Studie eingeholt (KEK-ZH-Nr.2013 - 0359).

\section{Messinstrumente}

Die dynamische Stabilisationsfähigkeit wurde mittels der Time-tostability (TTS, nach Colby et al. [22]) auf einer Kraftmessplatte (AMTI Accusway Plus $\left.{ }^{\circledR}, 2008\right)$ analysiert. Mithilfe der TTS ist es möglich, die neuromuskuläre Kontrolle zu messen, welche sowohl das sensorische als auch das motorische System während einer komplexen Bewegung wie der Landung nach einem Sprung erfasste 
[3]. Dabei wurde der Verlauf des Körperschwerpunktes während und nach der Landung aufgezeichnet. Die TTS erfasst die Fähigkeit des Körpers, Schwankungen beim Übergang vom dynamischen in den statischen Zustand zu minimieren [3, 23]. Die Datenerfassung erfolgte durch die SWAYWIN95 ${ }^{\circledR}$ Software. Mit einer Abtastrate von $50 \mathrm{~Hz}$ wurden die Kräfte in den drei Raumrichtungen (anteroposterior, mediolateral, vertikal) mit dem Accusway Plus System gemessen. Mit der Formel von Colby et al. (1999) wurde mittels MATLAB $^{\circledR}$ die TTS in anteroposteriore (AP) und mediolaterale (ML) Richtung berechnet. Die Landung wurde definiert als der Punkt des maximalen Ausschlages in vertikaler Richtung.

Für die Messung der Oberflächentemperatur wurde ein LUX Tools ${ }^{\circledR}$ LDT-6 Lasertemperaturmesser (2011) eingesetzt.

\section{Intervention}

Als Kühlspray wurde Perskindol ${ }^{\circledR}$ Cool Spray verwendet (1 g enthält $5 \mathrm{mg}$ Levomenthol als Wirkstoff, als Treibgas Propan/Butan, Dimethylether, Aromatica, Hilfsstoffe). Als Wasserspray verwendeten wir einen handelsüblichen mechanischen Sprühspray gefüllt mit raumtemperiertem Wasser.

\section{Tests}

Test 1: Side-Step-down

Der Proband wurde aufgefordert, barfuss auf eine $25 \mathrm{~cm}$ hohe Stufe zu stehen, die seitlich zur Messplatte ausgerichtet war ( $\bullet$ Abb. 1a). Die Innenseite des Standbeines wurde bündig zur Kante der Stufe ausgerichtet, das unbelastete Testbein schaute seitlich über die Stufe hinaus ( $\triangle$ Abb. 1b). Zwischen der Stufe und der Kraftmessplatte befand sich eine $15 \mathrm{~cm}$ breite Holzleiste, die Landefläche war auf der Kraftmessplatte markiert ( $\bullet$ Abb. 1a). Die Hände hatte der Proband während der gesamten Testdurchführung seitlich am Beckenkamm zu platzieren. Er wurde aufgefordert, einen Schritt zur Seite zu machen und mit dem Testbein innerhalb der Markierung zu landen. Bei der Landung sollte der Proband so schnell als möglich still stehen und die Position bis zum Ertönen des Signals (ca. $10 \mathrm{~s}$ ) halten ( $\triangle$ Abb. 1c).

\section{Test 2: 1-Leg-Jump}

Der Proband wurde aufgefordert, sich barfuss vor der Kraftmessplatte zu platzieren ( $\bullet$ Abb. 2b). Der Abstand zur markierten Landefläche entsprach der individuellen Distanz vom lateralen Malleolus zum Trochanter major [22]. Der Proband führte einen einbeinigen Sprung aus, wobei er nach der Landung so schnell als möglich still stehen und die Position bis zum Ertönen des Signals halten sollte ( $\bullet$ Abb. $2 \mathbf{c})$.

\section{Testablauf}

Die rekrutierten Probanden erhielten eine Nummer und füllten einen anonymisierten Fragebogen zum Erfassen der Kovariablen Alter, Größe, Gewicht, Geschlecht, Vorverletzungen, dominantes Bein [24] und TAS aus. Sie wurden nach drei Kriterien stratifiziert randomisiert (TAS, Geschlecht und Vorverletzungen des OSG)

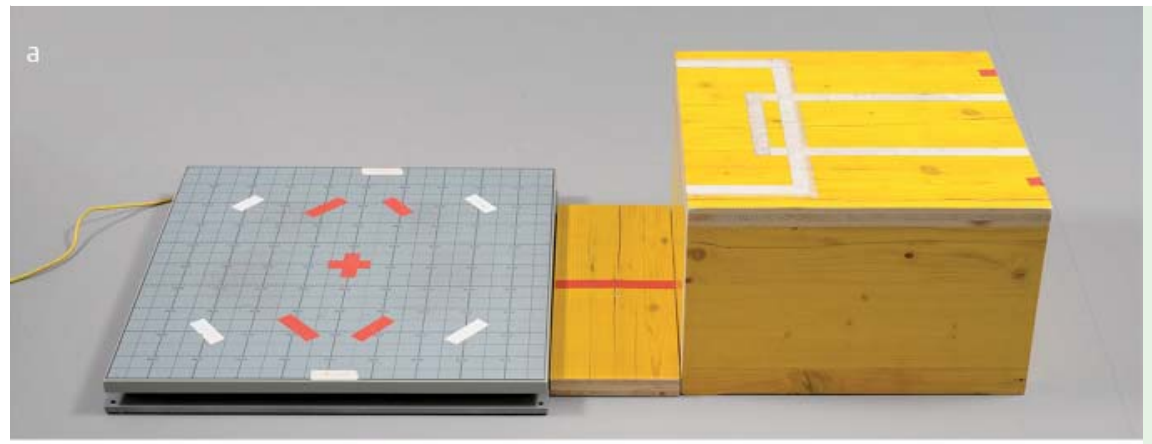

Abb. 1 Side-Step-down. a Installation. b Ausgangsstellung. $\mathbf{c}$ Endstellung.

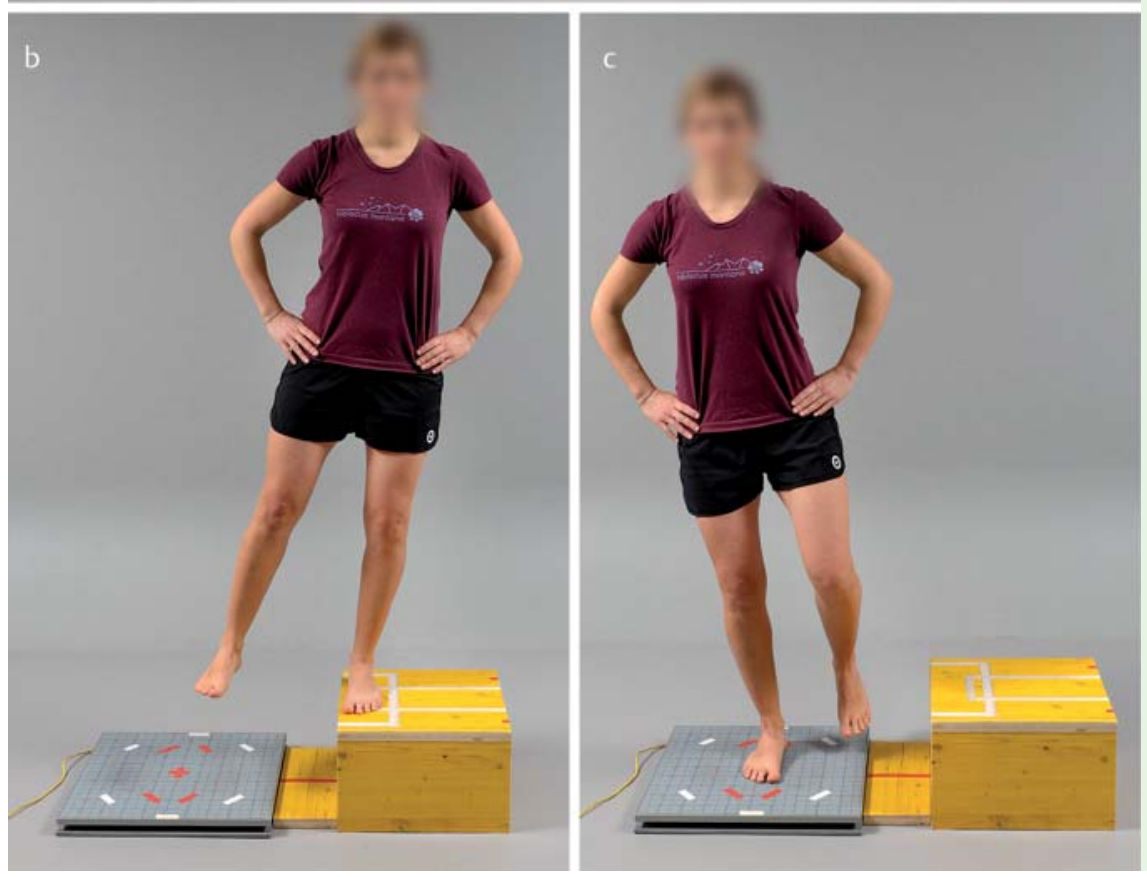




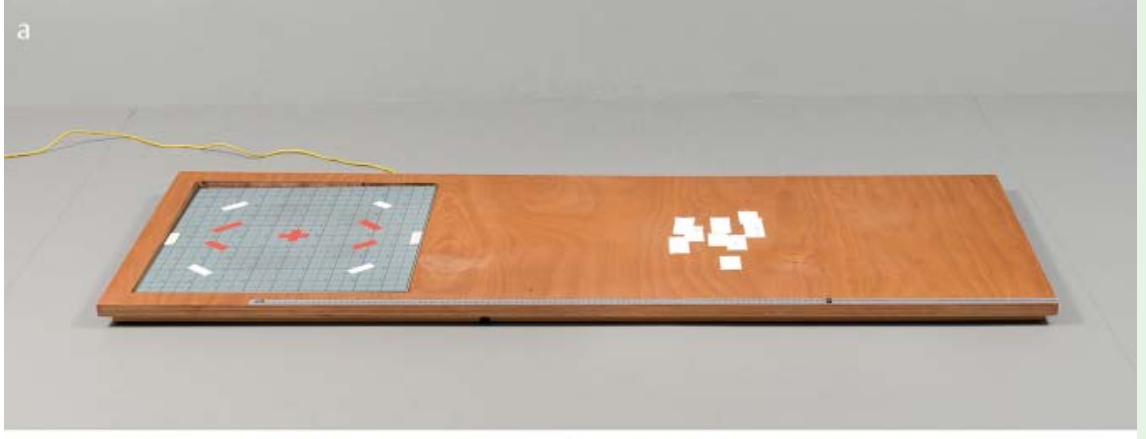

Abb. 2 1-Leg-Jump. a Installation. b Ausgangsstellung. $\mathbf{c}$ Endstellung.
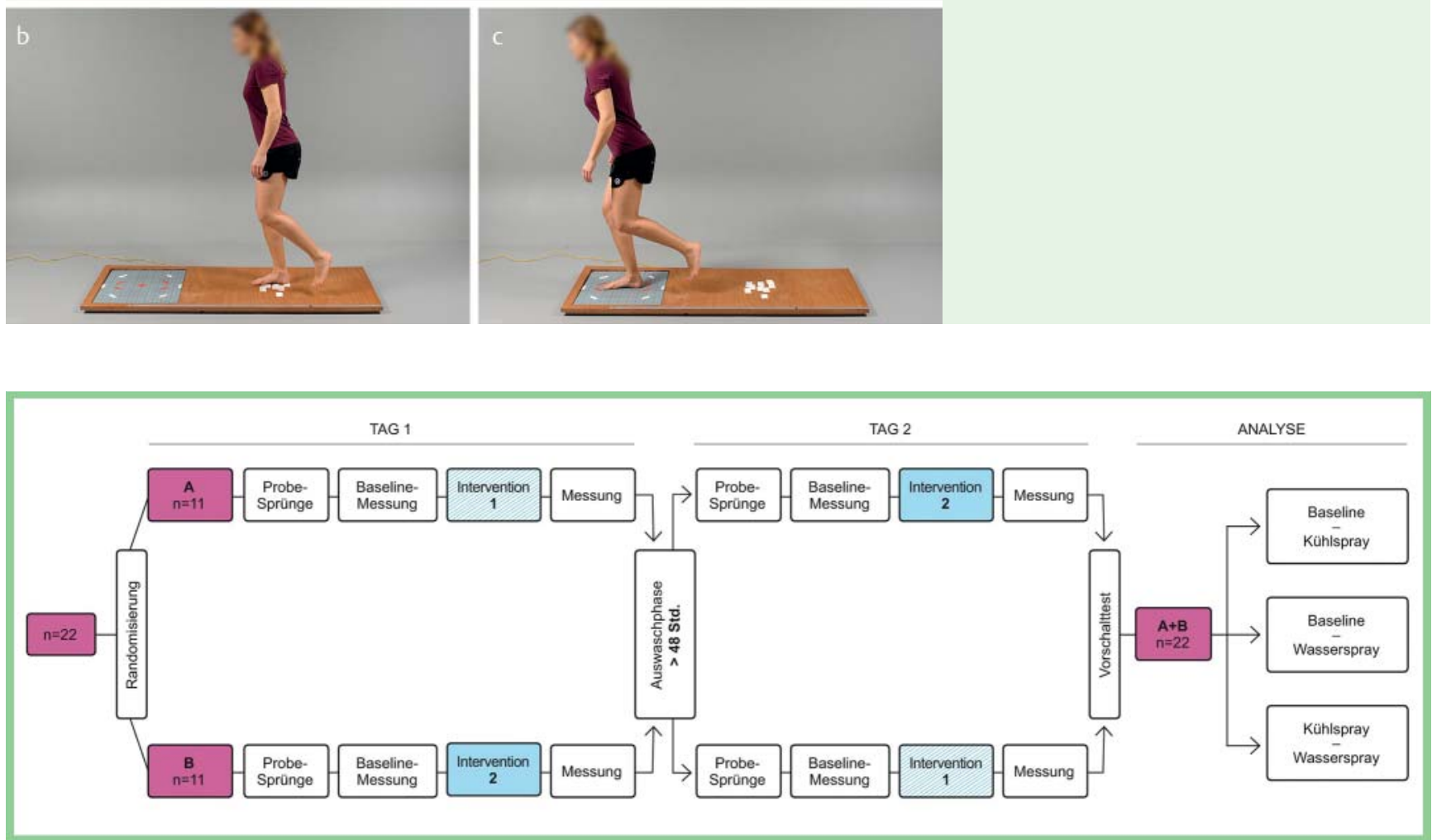

Abb. 3 Flussdiagramm Studienablauf.

und zwei Gruppen (A und B) zugeteilt. Die Randomisierung wurde durch eine unbeteiligte Person durchgeführt.

Alle Tests wurden im gleichen Raum von jeweils einem von drei geschulten Testern durchgeführt. Die Beurteilung der dynamischen Stabilisationsfähigkeit erfolgte an zwei Testtagen mit mindestens 48 Stunden Pause.

Am ersten Testtag wärmte sich der Proband fünf Minuten locker laufend auf dem Laufband auf, bevor ihm die Sprünge mündlich instruiert und vorgezeigt wurden. Danach wurde er aufgefordert, pro Bein 3 Probesprünge des Side-Step-downs und 5 Probesprünge des 1-Leg-jumps auszuführen, um einen Lerneffekt während der Tests zu minimieren [22]. Anschließend wurde sitzend eine 5-minütige Pause eingelegt.

Es erfolgte eine Baseline-Messung ohne Intervention. Der Proband startete jeweils mit dem dominanten Bein und führte seitenalternierend je 3 Side-Step-downs und 3 1-Leg-jumps aus. Verloren die Probanden während des Sprunges das Gleichgewicht oder berührten mit dem gegenüberliegenden Fuss den Boden, wurde der Sprung abgebrochen und wiederholt. Anschließend folgte sitzend eine 5-minütige Pause.
Danach folgte die Interventionsmessung. Probanden der Gruppe A starteten mit Intervention 1 (Kühlspray am dominanten und Wasserspray am nicht dominanten Fuß). Probanden der Gruppe B starteten mit Intervention 2 (Wasserspray am dominanten und Kühlspray am nicht dominanten Fuß) ( $\bullet$ Abb.3). Die Probanden wurden nicht darüber informiert, welche Intervention an welchem Fuss appliziert wurde, die Spraydosen waren mit weißer Folie abgeklebt. Die Applikation des Kühlsprays erfolgte aus 15,5 cm Distanz mit zehn Sprühstößen (ca. $1 \mathrm{~s}$ ) kaudal des lateralen Malleolus im Bereich des Ligamentum collaterale laterale. Die Applikation des Wassersprays erfolgte ebenfalls aus 15,5 cm Distanz mit drei Sprühstößen über demselben Bereich. Vor jedem Sprung wurde die Applikation wiederholt. Die Reihenfolge der Sprünge fand seitenalternierend statt. Es wurden je 3 Side-Stepdowns und 3 1-Leg-jumps ausgeführt.

Am Testtag 2 wurde das Protokoll vom Testtag 1 mit vertauschten Interventionen wiederholt. Gruppe A erhielt Intervention 2, Gruppe B Intervention 1 ( $\bullet$ Abb. 3). Die Oberflächentemperatur am lateralen OSG wurde stichprobenartig während der gesamten Testreihe erfasst und überprüft. 


\section{Datenanalyse und Statistik}

Mit der deskriptiven Statistik wurden die demografischen Variablen und die Oberflächentemperatur der Probanden beschrieben. Von den jeweils drei Messungen des Side-Step-down und des 1-Leg-jump wurde der Mittelwert berechnet. Die Anwendung des Shapiro-Wilk-Tests erfolgte um zu überprüfen, ob eine Normalverteilung vorlag.

Der Einfluss der Zusatzvariablen (Alter, Geschlecht, Größe, Gewicht, Vorverletzung des OSG, TAS, dominantes Bein) auf die TTS-Ergebnisse wurde mit einer linearen Regression geprüft. Falls eine Zusatzvariable einen Einfluss auf die TTS hatte, wurde das Ergebnis separat untersucht.

Mit dem Mann-Whitney-U-Test (Vorschalttest) wurde das Vorliegen eines Überhangeffektes geprüft. Waren keine Überhangeffekte vom ersten zum zweiten Testtag vorhanden, konnte der Einfluss der Interventionen Kühlspray und Wasserspray auf die TTS sowie der Unterschied zwischen den beiden Interventionen selbst mit einem Wilcoxon-Rank-Test untersucht werden. Die gesamten Daten wurden im IBM-SPSS $21^{\circledR}$ (PASW Statistics, Chicago, IL) Statistikprogramm gesammelt, gespeichert und analysiert. Das Signifikanzniveau wurde bei $\mathrm{p} \leq 0,05$ definiert.

\section{Ergebnisse}

\section{$\nabla$}

\section{Deskriptive Statistik}

Für die Studie wurden 22 Teilnehmer (15 Frauen, 7 Männer) mit einem Durchschnittsalter von $31,8 \pm 5,7$ Jahren rekrutiert. Bei den demografischen Daten war kein Gruppenunterschied vorhanden ( $\bullet$ Tab. 1). Die Oberflächentemperatur vor Intervention lag bei $30,5^{\circ} \mathrm{C}$, nach Kühlsprayapplikation war sie im Schnitt $11,5^{\circ} \mathrm{C}$ kälter, nach Wassersprayapplikation $3,7^{\circ} \mathrm{C}$. Keine der Messungen unterschritt $13^{\circ} \mathrm{C}$. Der Shapiro-Wilk-Test zeigte, dass bei keiner der Variablen eine Normalverteilung vorlag.

\section{Zusammenhang TTS und Intervention}

Der Vorschalttest zeigte keine Überhangseffekte vom Testtag 1 zum Testtag 2 an ( $\bullet$ Tab.2).

Bei der Analyse mit dem Wilcoxon-Rank-Test wurde keine signifikante Veränderung der TTS festgestellt, weder nach der Kühlspray- respektive der Wassersprayintervention noch zwischen den beiden Interventionen selber.Einzig beim Side-Step-down nach der Applikation von Wasserspray am dominanten Bein zeigt sich eine signifikante Veränderung der TTS in mediolateraler Richtung. Mittelwert der Baseline-Messung betrug 1,65 Sekunden, nach der Applikation von Wasserspray waren die Probanden bei einem Mittelwert von 1,70 Sekunden um 0,05 Sekunden langsamer $(\mathrm{p}<0,05$, SD 0,11). Nach der Applikation von Kühlspray zeigte sich keine Veränderung der TTS ( $\bullet$ Tab. 3 ).

\section{Zusatzvariablen}

Die Überprüfung der Zusatzvariablen mittels einer linearen Regression zeigte einen signifikanten Einfluss der Variablen „Vorverletzung des OSG" nach Kühlsprayapplikation am nicht dominanten Bein auf die TTS in mediolaterale Richtung beim 1-Legjump $\left(R^{2}=0,22, F(21 / 2)=5,693, p=0,027\right)$. Am dominanten Bein war keine Signifikanz, jedoch eine Tendenz sichtbar $\left(R^{2}=0,16\right.$, $F(21 / 2)=3,893, p=0,062)$. Es wurden keine weiteren Einflüsse der Zusatzvariablen auf die TTS-Resultate entdeckt. Die weitere Analyse der Zusatzvariablen „Vorverletzung des OSG“ konnte nicht bestätigt werden ( $\odot$ Tab. 4 ).
Tab. 1 Deskriptive Statistik.

\begin{tabular}{|c|c|c|c|c|c|c|}
\hline & \multicolumn{2}{|l|}{ alle } & \multicolumn{2}{|c|}{ Gruppe A } & \multicolumn{2}{|c|}{ Gruppe B } \\
\hline & $\begin{array}{l}\text { Mittel- } \\
\text { wert }\end{array}$ & $S^{1}$ & $\begin{array}{l}\text { Mittel- } \\
\text { wert }\end{array}$ & SD & $\begin{array}{l}\text { Mittel- } \\
\text { wert }\end{array}$ & SD \\
\hline Alter (Jahre) & 31,8 & 5,7 & 32,7 & 5,0 & 30,9 & 6,5 \\
\hline Größe $(\mathrm{cm})$ & 172,8 & 7,0 & 172,4 & 7,3 & 173,3 & 7,0 \\
\hline Gewicht (kg) & 66,9 & 8,3 & 65,1 & 9,0 & 68,6 & 7,5 \\
\hline Geschlecht (w/m) & $15 / 7$ & & $8 / 3$ & & $7 / 4$ & \\
\hline $\begin{array}{l}\text { Vorverletzungen des } \\
\text { OSG (ja/nein) }\end{array}$ & $12 / 10$ & & $6 / 5$ & & $6 / 5$ & \\
\hline $\mathrm{TAS}^{2}$ Median (min./max.) & $5(4 / 7)$ & & $5(4 / 7)$ & & $5(4 / 7)$ & \\
\hline
\end{tabular}

1 Standard Deviation.

${ }^{2}$ Tegner-Aktivitätsskala.

Tab. 2 Test nach Überhangeffekt bei den Baseline-Messungen der beiden Crossover-Gruppen A und B.

\begin{tabular}{|c|c|c|c|c|c|c|c|}
\hline & & & \multicolumn{2}{|c|}{$\begin{array}{l}\text { Gruppe A } \\
n=11\end{array}$} & \multicolumn{2}{|c|}{$\begin{array}{l}\text { Gruppe B } \\
n=11\end{array}$} & \multirow{2}{*}{$\begin{array}{l}\text { Mann- } \\
\text { Whitney- } \\
\text { U-Test } \\
\text { p-Wert }\end{array}$} \\
\hline & & & $\begin{array}{l}\text { Mittel- } \\
\text { wert }\end{array}$ & SD & $\begin{array}{l}\text { Mittel- } \\
\text { wert }\end{array}$ & SD & \\
\hline \multirow{4}{*}{$\begin{array}{l}\text { Side-Step- } \\
\text { down; (s) }\end{array}$} & \multirow[t]{2}{*}{$\mathrm{AP}^{1}$} & $D^{2}$ & 1,907 & 0,069 & 1,925 & 0,043 & 0,748 \\
\hline & & $N^{3}$ & 1,905 & 0,048 & 1,924 & 0,044 & 0,438 \\
\hline & \multirow[t]{2}{*}{$\mathrm{ML}^{4}$} & D & 1,639 & 0,095 & 1,659 & 0,113 & 0,652 \\
\hline & & ND & 1,716 & 0,051 & 1,668 & 0,105 & 0,217 \\
\hline \multirow{4}{*}{$\begin{array}{l}\text { 1-Leg- } \\
\text { Jump; (s) }\end{array}$} & \multirow[t]{2}{*}{ AP } & D & 1,854 & 0,228 & 1,794 & 0,226 & 0,401 \\
\hline & & ND & 1,702 & 0,244 & 1,704 & 0,291 & 0,949 \\
\hline & \multirow[t]{2}{*}{$\mathrm{ML}$} & D & 1,743 & 0,049 & 1,776 & 0,024 & 0,133 \\
\hline & & ND & 1,753 & 0,055 & 1,760 & 0,043 & 0,847 \\
\hline
\end{tabular}

${ }^{1}$ Anteroposteriore Richtung.

2 Dominantes Bein.

${ }^{3}$ Nicht dominantes Bein.

${ }^{4}$ Mediolaterale Richtung.

\section{Diskussion}

Soweit uns bekannt, ist dies die erste Studie, welche den Effekt von Kühlspray am OSG auf die dynamische Stabilisationsfähigkeit untersucht.

Bei Probanden mit einer früheren Verletzung des OSG wurde die Zeit bis zur dynamischen Stabilisation in mediolaterale Richtung am nicht dominanten Bein signifikant verlängert. Am dominanten Bein zeigte sich keine Signifikanz, es war jedoch eine Tendenz sichtbar. Mögliche Einflüsse könnten die lokale, mechanische Stabilität des OSG [25], die funktionelle Stabilität [13, 25] sowie eine zentrale Komponente einschließlich Angst und Kinesiophobie [26] aufgrund vorgängiger Verletzungen sein. Vergleichbar damit sind die Resultate von Kernozek et al. [13] die zeigen, dass Probanden mit einer frischen Grad I-Verletzung des lateralen OSG eine verminderte statische Stabilisationsfähigkeit im Einbeinstand in mediolaterale Richtung aufweisen im Vergleich zur gesunden Extremität. Nach einem 20-minütigen Eisbad in $4^{\circ} \mathrm{Cel}-$ sius kaltem Wasser war dieser Unterschied noch deutlicher. Douglas et al. [14] zeigten nach einem 15-minütigen Eisbad bei gleicher Temperatur eine verminderte mediolaterale Stabilisationsfähigkeit bei gesunden Probanden. Der Test erfolgte im Einbeinstand auf einer sich bewegenden Unterlage. Zu bedenken ist, dass der mediolateralen Stabilität vor allem beim Verletzungsmechanismus der OSG-Distorsion eine wichtige Rolle zukommt 
Tab. 3 Veränderung der TTS nach Wasserspray- und Kühlsprayapplikation.

\begin{tabular}{|c|c|c|c|c|c|c|c|c|c|c|c|}
\hline & & & Baseline & & Wasserspra & & $\mathrm{Z}^{1}$ & Kühlspray & & $Z^{2}$ & $\mathrm{Z}^{3}$ \\
\hline & & & Mittelwert & SD & Mittelwert & SD & p-Wert & Mittelwert & SD & p-Wert & p-Wert \\
\hline \multirow{4}{*}{$\begin{array}{l}\text { Side-Step- } \\
\text { down; } \\
n=22(s)\end{array}$} & \multirow[t]{2}{*}{$\mathrm{AP}^{4}$} & $D^{5}$ & 1,916 & 0,057 & 1,894 & 0,047 & 0,135 & 1,893 & 0,039 & 0,108 & 1,000 \\
\hline & & $\mathrm{ND}^{6}$ & 1,914 & 0,046 & 1,910 & 0,064 & 0,711 & 1,901 & 0,090 & 0,548 & 0,614 \\
\hline & \multirow[t]{2}{*}{$\mathrm{ML}^{7}$} & $\mathrm{D}$ & 1,649 & 0,102 & 1,695 & 0,106 & 0,016 & 1,657 & 0,110 & 0,709 & 0,079 \\
\hline & & ND & 1,692 & 0,084 & 1,670 & 0,110 & 0,394 & 1,674 & 0,156 & 0,404 & 0,758 \\
\hline \multirow{4}{*}{$\begin{array}{l}\text { 1-Leg- } \\
\text { Jump; } \\
\mathrm{n}=22 \text { (s) }\end{array}$} & \multirow[t]{2}{*}{ AP } & $\mathrm{D}$ & 1.823 & 0,224 & 1,837 & 0,186 & 0,715 & 1,819 & 0,191 & 0,917 & 0,897 \\
\hline & & ND & 1,703 & 0,262 & 1,778 & 0,185 & 0,178 & 1,715 & 0,198 & 0,721 & 0,153 \\
\hline & \multirow[t]{2}{*}{$\mathrm{ML}$} & $\mathrm{D}$ & 1,760 & 0,042 & 1,766 & 0,048 & 0,495 & 1,759 & 0,027 & 0,615 & 0,291 \\
\hline & & ND & 1,757 & 0,048 & 1,767 & 0,043 & 0,211 & 1,770 & 0,055 & 0,229 & 0,944 \\
\hline
\end{tabular}

${ }^{1}$ Wilcoxon-Rank-Test, Vergleich Baseline - Wasserspray.

${ }^{2}$ Wilcoxon-Rank-Test, Vergleich Baseline - Kühlspray.

${ }^{3}$ Wilcoxon-Rank-Test, Vergleich Wasserspray - Kühlspray.

${ }^{4}$ Anteroposteriore Richtung.

5 Dominantes Bein.

${ }^{6}$ Nicht dominantes Bein.

${ }^{7}$ Mediolaterale Richtung.

\begin{tabular}{|lllllllllll|}
\hline & $\begin{array}{l}\text { Landung } \\
\text { auf Bein }\end{array}$ & Baseline & & Wasserspray & Kühlspray & $\mathbf{Z}^{1}$ & $\mathbf{Z}^{2}$ \\
\hline $\begin{array}{l}\text { 1-Leg-Jump } \\
\mathbf{M L}^{3}\end{array}$ & & $\begin{array}{l}\text { Mittel- } \\
\text { wert }\end{array}$ & SD & $\begin{array}{l}\text { Mittel- } \\
\text { wert }\end{array}$ & SD & $\begin{array}{l}\text { Mittel- } \\
\text { wert }\end{array}$ & SD & p-Wert & p-Wert \\
\hline $\begin{array}{l}\text { gesund } n=12 \\
(s)\end{array}$ & ND $^{4}$ & 1,748 & 0,047 & 1,764 & 0,037 & 1,747 & 0,056 & 0,844 & 0,108 \\
\hline $\begin{array}{l}\text { vorverletzt bds } \\
n=6(s)\end{array}$ & ND & 1,778 & 0,050 & 1,761 & 0,064 & 1,792 & 0,049 & 0,276 & 0,462 \\
\hline $\begin{array}{l}\text { vorverletzt re } \\
n=4(s)\end{array}$ & ND & 1,738 & 0,039 & 1,778 & 0,024 & 1,798 & 0,044 & 0,144 & 0,465 \\
\hline
\end{tabular}

Tab. 4 Veränderung der TTS beim 1-Leg-Jump-Test in mediolaterale Richtung nach Wasserspray- und Kühlsprayapplikation bei gesunden und vorverletzten Probanden.

${ }^{1}$ Wilcoxon-Rank-Test, Vergleich Baseline - Kühlspray.

${ }^{2}$ Wilcoxon-Rank-Test, Vergleich Wasserspray - Kühlspray.

${ }^{3}$ Mediolaterale Richtung.

${ }^{4}$ Nicht dominantes Bein.

[19]. Möglicherweise fallen die Ergebnisse bei akut verletzten Athleten anders aus, welche nach der Kühlsprayapplikation ins Spiel zurückkehren würden [13].

Es wurden keine weiteren signifikanten Einflüsse der Kühlspraybeziehungsweise Wassersprayinterventionen auf die TTS gefunden. Eine Ausnahme macht der Side-Step-down des dominanten Beines in mediolaterale Richtung nach der Applikation von Wasserspray. Da unsere Resultate keine weiteren signifikanten Ergebnisse nach Wasserspray beziehungsweise Side-Step-down zeigen, gehen wir von einem zufälligen Resultat aus ( $\bullet$ Tab. 3).

Die Messwerte der TTS sind vergleichbar mit denjenigen von Colby et al. [22]. Im Gegensatz zu ihm fanden wir keinen signifikanten Unterschied der TTS zwischen dominantem und nicht dominantem Bein. Dies wurde möglicherweise durch unsere mehrheitlich aus Physiotherapeuten bestehende Probandengruppe (18/22) beeinflusst. Therapeuten sind sich berufsbedingt an stabilisierende Übungen gewohnt und führen beim Vorzeigen im Therapiesetting diese Art von Sprüngen regelmäßig aus, was zu einem möglichen Trainingseffekt des nicht dominanten Beines führen kann. Weiter stellt sich die Frage, ob die Testauswahl wie bei Colby et al. [22] verwendet, zu einfach gewählt wurde oder, ob eine Vorermüdung der Probanden das Resultat signifikant beeinflusst hätte.

Mit einer durchschnittlichen Ausgangstemperatur der Hautoberfläche von $31,5^{\circ} \mathrm{C}$ decken sich unsere Resultate mit denjenigen von Dykstra et al. [27]. Ausgehend von $31^{\circ} \mathrm{C}$ untersuchten sie die oberflächliche sowie die intramuskuläre Temperatur nach 20-minütiger Kälteapplikation der drei Kühlanwendungen Eiswürfel, zerkleinertes Eis und schmelzendes Eis. Sie erreichten Oberflächentemperaturen von zwischen $17^{\circ} \mathrm{C}$ (Eiswürfel), $16^{\circ} \mathrm{C}$ (zerstoßenes Eis) und $14{ }^{\circ} \mathrm{C}$ (schmelzendes Eis). Mit einer durchschnittlichen Reduktion der Oberflächentemperatur von $10^{\circ} \mathrm{C}$ nach der Kühlsprayapplikation auf durchschnittlich $20,1^{\circ} \mathrm{C}$ lagen wir deutlich über den Werten von Dyksta et al. [27]. In einzelnen Fällen erreichte die Kühlsprayapplikation durch einen kumulierten Effekt vor dem 3. Sprung Werte bis unter $14{ }^{\circ} \mathrm{C}$, welche aber immer noch über dem Schwellenwert für eine analgetische wie auch neurale Reaktion von $13^{\circ} \mathrm{C}$ lagen $[5,7]$. Auch wenn der 3. Sprung des Side-Step-down und 1-Leg-Jump mit den Baseline-Werten verglichen wurde, führte die kumulative Wirkung vom Kühlspray auf das Gewebe zu keiner Veränderung in der TTS. Mit der kurzen vom Hersteller empfohlenen Applikationszeit lagen wir um ein vielfaches unter den Applikationszeiten oben genannter Studie und gehen nicht von einer Tiefenwirkung des Kühlsprays aus.

Weiter muss die relativ kleine verletzungsspezifische Applikationsfläche bedacht werden, die auf das laterale OSG im Bereich des Ligamentes collaterale laterale beschränkt war. Saam et al. [15] testeten den Einfluss einer 10-minütigen Eisbeutelapplikation am OSG auf das statische Gleichgewicht und fanden keine Evidenz für einen negativen Effekt. Sie formulierten die Hypothese, dass die Wahrnehmung dieser minimalen Gelenksbewegungen hauptsächlich über die intramuskulären Rezeptoren erfolgt 
und somit das statische Gleichgewicht durch eine auf das Gelenk beschränkte Kälteapplikation nicht beeinflusst wird, auch wenn möglicherweise die Feuerungsrate der Gelenksrezeptoren und die Leitgeschwindigkeit der dazugehörigen Nervenfasern durch die Kälteapplikation verändert sein sollte. Auch Williams et al. [12], welche die 20-minütige Applikation von zerstoßenem Eis auf das OSG beschränkten, fanden bei ihren Ergebnissen keine signifikanten Veränderungen bei statischen und dynamischen Aktivitäten. Sie bemerkten, dass der Einfluss von Kälte auf die Propriozeption bei Applikation auf das ein Gelenk umgebende Gewebe ein anderer ist als die Kühlung von Muskulatur. Die großflächige Kühlung des Kniegelenkes mit zerstoßenem Eis wie bei der Studie von Schmid et al. [16] führte nach 20 Minuten zu einer signifikanten Veränderung der elektromyografischen Aktivität der Muskulatur in der Voraktivierung, der exzentrischen sowie der konzentrischen Phase bei einem einbeinigen Drop-Jump.Dies zeigte sich direkt nach der Applikation wie auch 20 Minuten später, was auf eine durch die Kälteapplikation verminderte Aktivität des neuromuskulären Systems hindeutet. Möglicherweise wäre in unserer Studie durch die Applikation des Kühlsprays über der Muskulatur eine Veränderung in der TTS größer oder auch evident bei nicht vorverletzten Probanden, weil die Muskelspindeln zur Stabilisationsfähigkeit mehr beitragen als die Rezeptoren der Haut [28]. Unsere Applikationsweise jedoch simuliert den sportspezifischen Einsatz bei akuten Verletzungen des OSG.

Der von unseren Probanden beschriebene langanhaltende Kühleffekt erklären wir uns durch den Einfluss von Levomenthol als Inhaltsstoff des Sprays. Levomenthol bzw. Menthol besitzt unter anderem eine schmerzlindernde und kühlende Wirkung und führt über bestimmte chemische Rezeptoren (TRPM8) zu demselben Kältegefühl wie tiefe Temperaturen [29].

Limitationen der Studie sind das Fehlen einer genauen Differenzierung der passiven Stabilität der OSG der Probanden mit Vorverletzung [25]. Die Verletzung bzw. Instabilität wurde nur anamnestisch erfragt. Weiter war die Probandengruppe von 22 Personen trotz Crossoverdesign möglicherweise zu klein, um Effekte in unseren Zahlen ( $\bullet$ Tab.4) zu zeigen. Ein Bias der Studie ist eine durch das Kältegefühl und den Geruch vom Menthol erschwerte Verblindung der Probanden. Es ist anzunehmen, dass der Kühlspray als solcher erkannt wurde. Einen durch die nicht verblindeten Tester verursachten Bias konnte nicht ausgeschlossen werden.

\section{Schlussfolgerung}

Unsere Ergebnisse zeigen keinen direkten Effekt der Kühlsprayapplikation am lateralen OSG auf die dynamische Stabilisationsfähigkeit in zwei unterschiedlichen Testsituationen bei gesunden, sportlich aktiven Probanden. Eine frühere Verletzung des OSG jedoch hat einen potenziellen Einfluss auf die dynamische Stabilisationsfähigkeit nach der Applikation von Kühlspray. Ob weitere die Stabilisationsfähigkeit beeinflussende Faktoren wie Vorermüdung und Gegenspieler oder die Applikation auf die Muskulatur diesen Effekt vergrößern, sollte in einer weiteren Untersuchung mit einer größeren Probandengruppe differenziert werden.

\section{Danksagung}

Ich möchte mich bei Monika Dörig und Doris Keller für die Durchführung der Testungen, bei Sebastian Hugelshofer für die grafische Unterstützung und bei Katharina Flieger für die sprachliche Korrektur der Arbeit bedanken.

\section{Interessenkonflikt: Nein}

\section{Literatur}

1 Hootman JM, Dick R, Agel J. Epidemiology of collegiate injuries for 15 sports: summary and recommendations for injury prevention initiatives. J Athl Train 2007; 42 (2): 311 - 319

2 Guillo S, Bauer T, Lee JW et al. Consensus in chronic ankle instability: aetiology, assessment, surgical indications and place for arthroscopy. Orthop Traumatol Surg Res 2013; 99 (8): 411-419

3 Miniello S, Dover G, Powers $M$ et al. Lower leg cold immersion does not impair dynamic stability in healthy women. J Sports Rehab 2005; 14 : $234-247$

4 Hubbard TJ, Denegar CR. Does cryotherapy improve outcomes with soft tissue injury? J Athl Train 2014; 39 (3): $278-279$

5 Chesterton L, Foster N, Ross L. Skin tempertature response to cryotherapy. Arch Phys Med Rehabil 2002; 83 (4): 543 - 549

6 Bleakley CM, Costello JT, Glasgow PD. Should athletes return to sport after applying ice? A systematic review of the effect of local cooling on functional performance. Sports Med 2012; 42 (1): 69-87

7 Glasgow P. Eisanwendung in der Sportphysiotherapie. Effekte der Kryotherapie. Sportphysio 2013; 1 (1): $37-44$

8 Algafy $A A$, George KP. The effect of cryotherapy on nerve conduction velocity, pain threshold and pain tolerance. Br J Sports Med 2007; 41 (6): $365-369$

9 Costello JT, Donnelly AE. Cryotherapy and joint position sense in healthy participants: a systematic review. J Athl Train 2010; 45 (3): $306-$ 316

10 Surenkok $O$, Aytar A, Akman M. Cryotherapy impairs knee joint position sense and balance. Isokinet Exerc Sci 2008; 16: 69-73

11 Wassinger CA, Myers JB, Gatti JM et al. Proprioception and throwing accuracy in the dominant shoulder after cryotherapy. J Athl Train 2007; 42 (1): $84-89$

12 Williams EE, Miller SJ 3rd, Sebastianelli WJ et al. Comparative immediate functional outcomes among cryotherapeutic interventions at the ankle. Int J Sports Phys Ther 2013; 8 (6): 828-837

13 Kernozek TW, Greany JF, Anderson DR et al. The effect of immersion cryotherapy on medial-lateral postural sway variability in individuals with a lateral ankle sprain. Physiother Res Int 2008; 13 (2): 107-118

14 Douglas M, Bivens S, Pesterfield J et al. Immediate effects of cryotherapy on static and dynamic balance. Int J Sports Phys Ther 2013; 8 (1): 9-14

15 Saam F, Leidinger B, Tibesku C. Der Einfluss der Kryotherapie am Sprunggelenk auf das statische Gleichgewicht. Sportverletz Sportschaden 2008; 22: 45-51

16 Schmid S, Moffat M, Gutierrez GM. Effect of knee joint cooling on the electromyographic activity of lower extremity muscles during a plyometric exercise. J Electromyogr Kinesiol 2010; 20 (6): 1075-1081

17 Jameson AG, Kinzey SJ, Hallam JS. Lower-extremity-joint cryotherapy does not affect vertical ground-reaction forces during landing. J Sport Rehabil 2001; 10: 132 - 142

18 Fischer J, Van Lunen BL, Branch JD et al. Functional performance following an ice bag application to the hamstrings. J Strength Cond Res 2009; 23 (1): $44-50$

19 Andersen TE, Floerenes TW, Arnason A et al. Video analysis of the mechanisms for ankle injuries in football. Am J Sports Med 2004; 32 (1): 69-79

20 Tegner Y, Lysholm J. Rating systems in the evaluation of knee ligament injuries. Clin Orthop Relat Res 1985; 198: 43-49

21 Wirth B, Meier N, Koch PP et al. Development and evaluation of a German version of the Tegner activity scale for measuring outcome after anterior cruciate ligament injury. Sportverletz Sportschaden 2013; 27 (1): $21-27$

22 Colby SM, Hintermeister RA, Torry MR et al. Lower limb stability with ACL impairment. J Orthop Sports Phys Ther 1999; 29 (8): 444-451; discussion 452-454 
23 Wikstrom EA, Powers ME, Tillman MD. Dynamic stabilization time after isokinetic and functional fatigue. J Athl Train 2004; 39 (3): 247-253

24 Schneiders AG, Sullivan SJ, O'Malley KJ et al. A valid and reliable clinical determination of footedness. PM R 2010; 2 (9): 835-841

25 Gehring D, Faschian K, Lauter B et al. Mechanical instability destabilises the ankle joint directly in the ankle-sprain mechanism. Br J Sports Med 2014; 48 (5): 377-382

26 Clanton TO, Matheny LM, Jarvis HC et al. Return to play in athletes following ankle injuries. Sports Health 2012; 4 (6): 471 - 474
27 Dykstra JH, Hill HM, Miller MG et al. Comparisons of cubed ice, crushed ice and wetted ice on intramuscular and surface temperature changes. J Athl Train 2009; 44 (2): 136-141

28 Riemann BL, Lephart SM. The sensorimotor system, part I: the physiologic basis of functional joint stability. J Athl Train 2002; 37 (1): 71 - 79

29 Patel T, Ishiuji Y, Yosipovitch G. Menthol: a refreshing look at this ancient compound. J Am Acad Dermatol 2007; 57 (5): 873-878 